either as false negatives of the PCR or as false positives of MFC. We can thus conclude that the junction region of the $\mathrm{IgH}$ rearrangement in MM is stable and can be used as a target for MRD assessment by ASO RQ-PCR and more, also by deep-sequencing methods, as it constantly identifies the myeloma cells responsible for relapse. ${ }^{15}$

In conclusion, our results show that, in the dominant myeloma clone, the CDR3 region of IGH remains constant across all the stages of disease evolution. This major clone signature is not modified by clinical or biological changes in the disease nor under different treatment pressures; accordingly, it would thus be responsible for disease relapses and progression, and could be used as a MRD target. Assuming that the CDR3 region remains stable, the recently raised concept of clonal tiding in MM should not be interpreted as a poly/oligoclonal but subclonal. In summary, in MM tides can be subclonal, but the ocean remains monoclonal.

\section{CONFLICT OF INTEREST}

The authors declare no conflict of interest.

\section{ACKNOWLEDGEMENTS}

This work is partially supported by grants PS09/01450 and PI12/02311 from the Spanish 'Instituto de Salud Carlos III (ISCIII)', grants RD12/0036/0069 \& 0058 from 'Red Temática de Investigación Cooperativa en Cáncer (RTICC), Spanish Ministry of Economy and Competitiveness' \& European Regional Development Fund (ERDF) 'Una manera de hacer Europa', grant number HUS412A12-1 from the 'Consejería de Educación de la Junta de Castilla y León', and grant GCB-120981SAN from the 'Asociación Española Contra el Cáncer (AECC)'. We thank Alicia Antón, Montserrat Hernández Ruano and Rebeca Maldonado for technical support.

N Puig ${ }^{1,2,3}$, I Conde ${ }^{1,2,3}$, C Jiménez ${ }^{1,2,3}$, ME Sarasquete ${ }^{1,2,3}$ A Balanzategui ${ }^{1,2,3}$, M Alcoceba $^{1,2,3}$, J Quintero ${ }^{4}$ MC Chillón ${ }^{1,2,3}$, E Sebastián ${ }^{1,2,3}$, R Corral ${ }^{1,2,3}$, L Marín $^{1,2,3^{\prime}}$ NC Gutiérrez ${ }^{1,2,3}$, M-V Mateos ${ }^{1,2,3}$, M González-Díaz ${ }^{1,2,3^{\prime}}$ JF San-Miguel ${ }^{5}$ and R García-Sanz ${ }^{1,2,3}$

${ }^{1}$ Department of Hematology, University Hospital of Salamanca,

Salamanca, Spain;

${ }^{2}$ IBSAL, Salamanca, Spain;

${ }^{3}$ IBMCC (USAL-CSIC), Salamanca, Spain;

${ }^{4}$ Department of Hematology, Hospital Miguel Servet, Zaragoza,

Spain and

${ }^{5}$ Clínica Universidad de Navarra, Centro de Investigación Médica Aplicada (CIMA), Pamplona, Spain

E-mail: margondi@usal.es

\section{REFERENCES}

1 Nowell PC. The clonal evolution of tumor cell populations. Science 1976; 194: 23-28.

2 Hallek M, Bergsagel PL, Anderson KC. Multiple myeloma: increasing evidence for a multistep transformation process. Blood 1998; 91: 3-21.

3 López-Corral L, Sarasquete ME, Beà S, García-Sanz R, Mateos MV, Corchete LA et al. SNP-based mapping arrays reveal high genomic complexity in monoclonal gammopathies, from MGUS to myeloma status. Leukemia 2012; 26: 2521-2529.

4 Puig N, Sarasquete ME, Balanzategui A, Martínez J, Paiva B, García $\mathrm{H}$ et al. Critical evaluation of ASO RQ-PCR for minimal residual disease evaluation in multiple myeloma. A comparative analysis with flow cytometry. Leukemia 2014; 28: 391-397.

5 Keats JJ, Chesi M, Egan JB, Garbitt VM, Palmer SE, Braggio E et al. Clonal competition with alternating dominance in multiple myeloma. Blood 2012; 120: 1067-1076.

6 Van Dongen JJ, Langerak AW, Brüggemann M, Evans PA, Hummel M, Lavender FL et al. Design and standardization of PCR primers and protocols for detection of clonal immunoglobulin and T-cell receptor gene recombinations in suspect lymphoproliferations: report of the BIOMED-2 Concerted Action BMH4-CT98-3936. Leukemia 2013: 17: 2257-2317.

7 Bakkus MH, Heirman C, Van Riet I, Van Camp B, Thielemens K. Evidence that multiple myeloma Ig heavy chain VDJ genes contain somatic mutations but show no intraclonal variation. Blood 1992; 80: 2326-2335.

8 Zojer N, Ludwig $\mathrm{H}$, Fiegl M, Stevenson FK, Sahota SS. Patterns of somatic mutations in $\mathrm{VH}$ genes reveal pathways of clonal transformation from MGUS to multiple myeloma. Blood 2003; 101: 4137-4139.

9 Ralph QM, Brisco MJ, Joshua DE, Brown R, Gibson J, Morley AA. Advancement of multiple myeloma from diagnosis through plateau phase to progression does not involve a new B-cell clone: evidence from the Ig heavy chain gene. Blood 1993; 82: 202-206.

10 Kühnemund A, Liebisch P, Bauchmüller K, zur Hausen A, Veelken H, Wäsch R et al. 'Light chain escape-multiple myeloma'- an escape phenomenon from plateau phase: report of the largest patient series using LC-monitoring. J Cancer Res Clin Oncol 2009; 135: 477-484

11 Rosiñol L, Oriol A, Teruel Al, Hernández D, López-Jiménez J, de la Rubia J et al. Programa para el Estudio y la Terapéutica de las Hemopatías Malignas/ Grupo Español de Mieloma (PETHEMA/GEM) group. Superiority of bortezomib, thalidomide, and dexamethasone (VTD) as induction pretransplantation therapy in multiple myeloma: a randomized phase 3 PETHEMA/GEM study. Blood 2012; 120: 1589-1596.

12 Sarasquete ME, García-Sanz R, González D, Martínez J, Mateo G, Martínez P et al. Minimal residual disease monitoring in multiple myeloma: a comparison between allelic-specific oligonucleotide real-time quantitative polymerase chain reaction and flow cytometry. Haematologica 2005; 90: 1365-1372.

13 Beishuizen A, Hählen K, Hagemeijer A et al. Multiple rearranged immunoglobulin genes in childhood acute lymphoblastic leukemia of precursor B cell origin. Leukemia 1991; 5: 657-667.

14 Langlands K, Craig Jl, Anthony RS, Parker AC. Clonal selection in acute lymphoblastic leukaemia demonstrated by polymerase chain reaction analysis of immunoglobulin heavy chain and T-cell receptor delta chain rearrangements. Leukemia 1993; 7: 1066-1070.

15 Martínez-Lopez J, Lahuerta JJ, Pepin F, González M, Barrio S, Ayala R et al. Prognostic value of deep sequencing method for minimal residual disease detection in multiple myeloma. Blood 2014; 123: 3073-3079.

\title{
CDK9 inhibition by dinaciclib potently suppresses Mcl-1 to induce durable apoptotic responses in aggressive MYC-driven B-cell lymphoma in vivo
}

Leukemia (2015) 29, 1437-1441; doi:10.1038/leu.2015.10

MYC dysregulation confers a poor prognosis to diffuse large B-cell lymphoma (DLBCL), and effective therapeutic strategies are lacking in relapsed/refractory DLBCL, Burkitt lymphoma and intermediate forms. ${ }^{1,2}$ As a master transcriptional regulator, MYC recruits transcription complexes containing RNA polymerase II (Pol II) to facilitate effective transcriptional elongation of MYC gene targets. ${ }^{3}$ Pol II is fully activated by phosphorylation of a critical serine residue at position 2 within heptapeptide repeats in the carboxy-terminal domain (CTD), a function performed by the positive transcription elongation factor $b$ ( $\mathrm{P}-\mathrm{TEFb}$; comprising CDK9 and cyclin T1). ${ }^{4}$ It has been shown that MYC binds and 

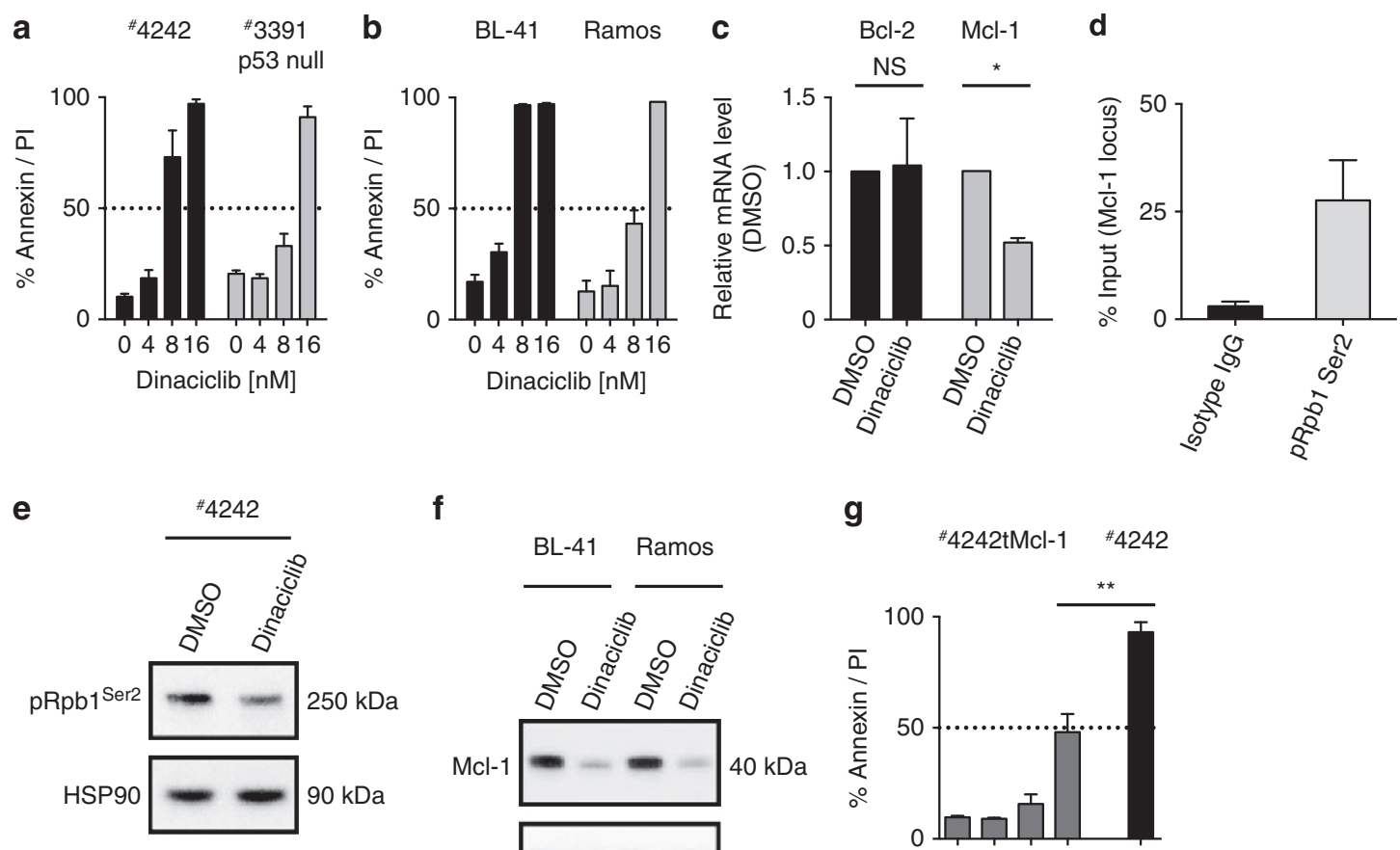

f

g BL-41 Ramos
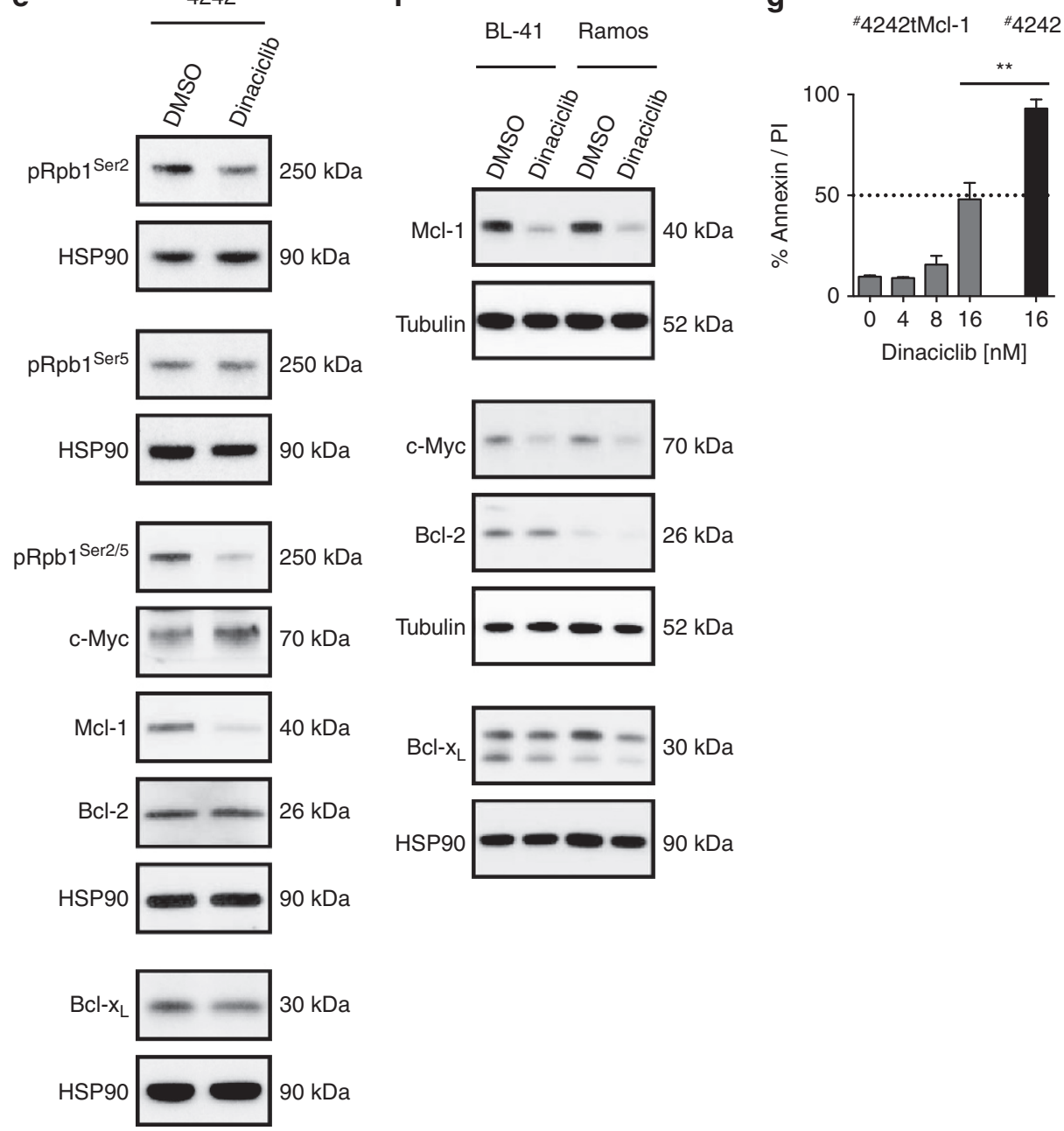

$250 \mathrm{kDa}$
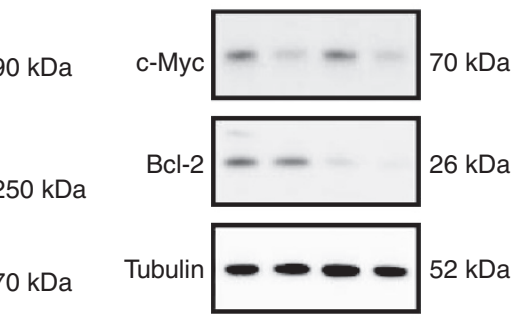

$40 \mathrm{kDa}$

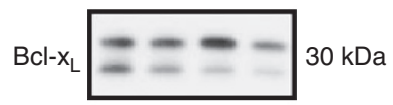

26 kDa

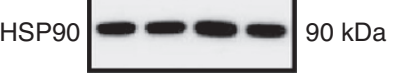

90 kDa

30 kDa

$90 \mathrm{kDa}$

Figure 1. Dinaciclib potently induces apoptosis of murine $E \mu-M y c$ and human IG-cMYC-translocated lymphomas with rapid and selective suppression of Mcl-1 transcription and protein levels. (a) Wild-type p53 ("4242) and p53-null ("3391) E with dimethylsulfoxide (DMSO) vehicle control or dinaciclib for $24 \mathrm{~h}$ and then analyzed using flow cytometric analysis for annexin-V/ propidium iodide (PI) positivity. (b) Human IG-cMYC-translocated BL-41 and Ramos cell lines were cultured in vitro with DMSO or dinaciclib for $48 \mathrm{~h}$ before the analysis of annexin-V/PI positivity using flow cytometry. (c) Mcl-1 and Bcl-2 mRNA expression in lymphoma \# 4242 following 3-h in vitro treatment with DMSO or $20 \mathrm{~nm}$ dinaciclib. Transcript levels are represented as fold change compared with DMSO. NS, not significant; ${ }^{*} P<0.0001$. (d) Chromatin immunoprecipitation-PCR of E $\mu-M y c$ lymphoma ${ }^{*} 4242$ cells showing binding of phospho-RNA Pol II CTD serine 2 (pRpb1 Ser2) at the Mcl-1 locus. Error bars denote the s.e.m. from three independent primer sets across the Mcl-1 locus. (e) E $\mu-M y c$ lymphoma \# 4242 was cultured in vitro for 3-h untreated or in the presence of DMSO or $20 \mathrm{~nm}$ dinaciclib before the preparation of lysates and immunoblotting for phospho-RNA Pol II CTD (pRpb1 ${ }^{\text {Ser2 }}, \mathrm{pRpb}^{\text {Ser5 }}$ and pRpb1 ${ }^{\text {Ser2/5 }}$ ), total Mcl-1, Bcl-2, Bcl- $x_{\mathrm{L}}$, C-MyC and HSP90 loading control. (f) Human IG-CMYC-translocated BL-41 and Ramos cell lines were cultured in vitro for $3 \mathrm{~h}$ in the presence of DMSO or $20 \mathrm{nM}$ dinaciclib before the preparation of lysates and immunoblotting for total $\mathrm{Mcl}-1, \mathrm{BCl}-2, \mathrm{BCl}-\mathrm{x}_{\mathrm{L}}, \mathrm{c}-\mathrm{Myc}$, Tubulin and HSP90 loading controls. (g) E $\mu-M y c$ lymphoma ${ }^{\#} 4242$ was transduced with murine stem cell virus expressing empty vector control or Mcl-1 and then cultured in vitro with dinaciclib for $24 \mathrm{~h}$ before flow cytometric analysis for annexin-V/PI positivity. ${ }^{* *} P<0.01$ comparing treatments at $16 \mathrm{~nm}$ concentration. All graphs represent the mean \pm s.e.m (error bars) for three or more independent experiments. 
a

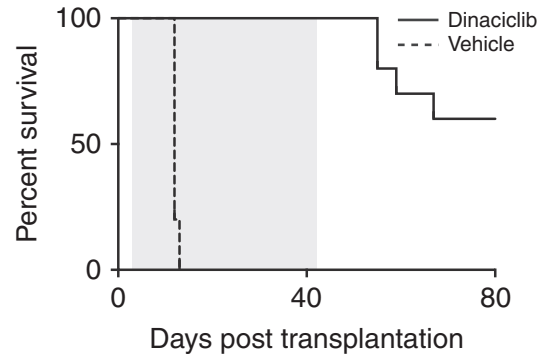

d

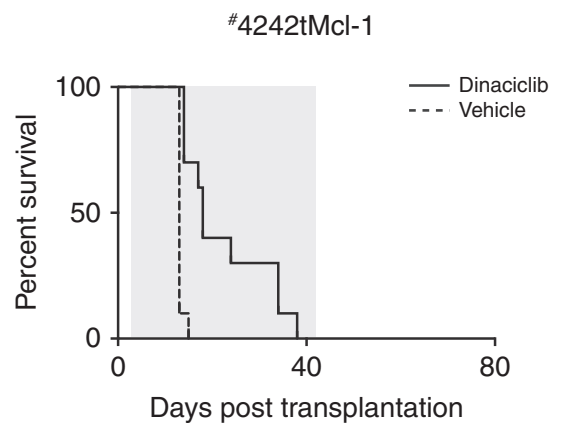

b

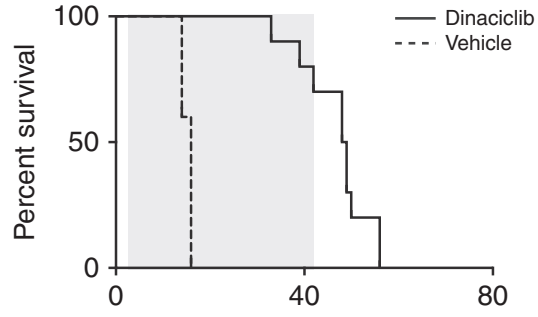

Days post transplantation

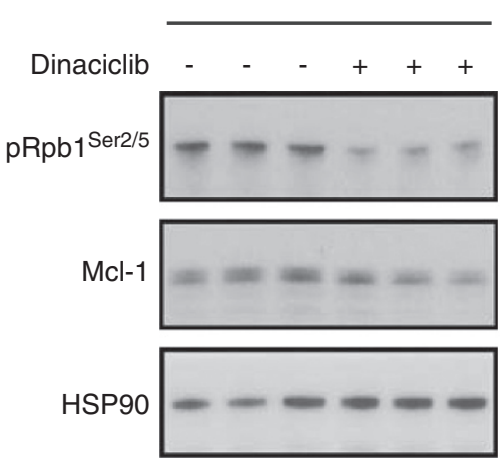

c \#106 dn p53

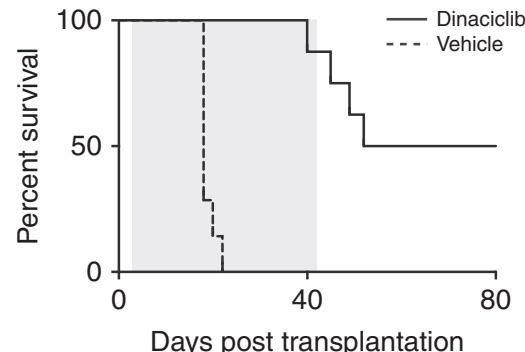

$4 \mathrm{~h}$

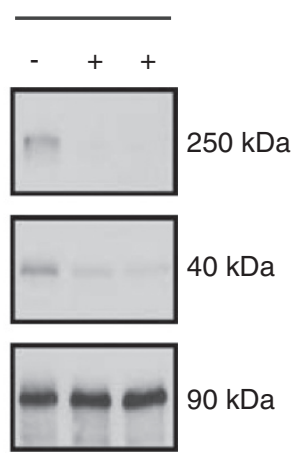

f
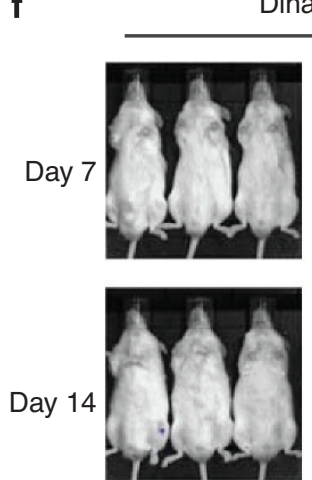

Dinaciclib
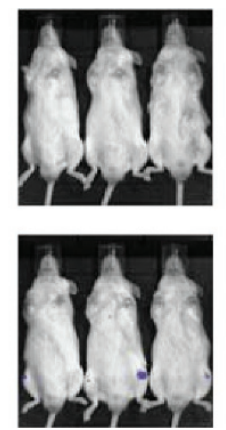

Vehicle
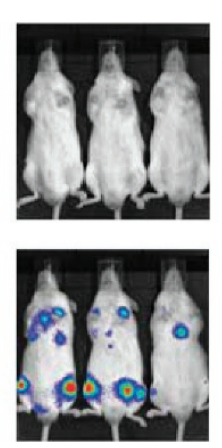

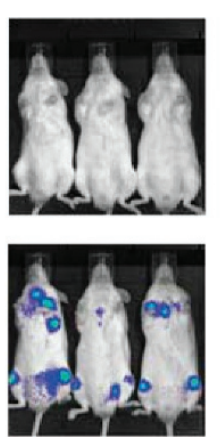

g

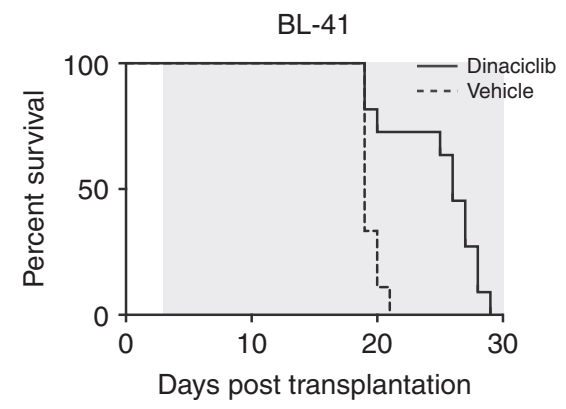

Figure 2. Dinaciclib therapy prolongs the survival of mice bearing E $\mu-M y c$ and human IG-cMYC-translocated lymphomas. (a-d) Kaplan-Meier survival curves representing cohorts of $\mathrm{C} 57 \mathrm{BI} / 6$ mice transplanted with representative $\mathrm{E} \mu-M y c$ lymphomas 3 days before the therapy commencement with $20 \%$ hydroxypropyl-beta-cyclodextran (HPBCD) vehicle or $30 \mathrm{mg} / \mathrm{kg}$ dinaciclib by intraperitoneal injection twice weekly. Gray shading denotes the period of therapy. dn, dominant negative; $P<0.0001$ for each experiment. The median survival for vehicle- and dinaciclib-treated mice were 12 days and not reached (" 4242$), 16$ and 48 days ( $\left.{ }^{*} 3391\right), 18$ and 66 days ( $\left.{ }^{\#} 106\right)$ and 13 and 18 days ( ${ }^{\#} 4242$ tMcl-1), respectively. (e) Lymph nodes were harvested from cohorts of C57BI/6 mice 1 or $4 \mathrm{~h}$ following a single dose of dinaciclib or $20 \% \mathrm{HPBCD}$,

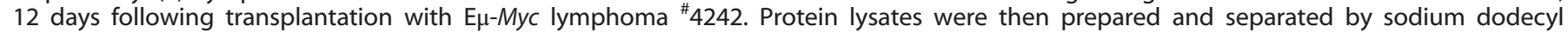
sulfate-polyacrylamide gel electrophoresis before immunoblotting for the indicated targets. Each lane represents protein lysate from the lymph nodes of an individual mouse. (f) Bioluminescence imaging of NOD-scid IL2R $\gamma^{\text {null }}$ mice transplanted with human IG-CMYC-translocated BL-41.luc2 lymphoma 3 days before the commencement of the therapy with $20 \%$ HPBCD vehicle or $30 \mathrm{mg} / \mathrm{kg}$ dinaciclib by intraperitoneal injection twice weekly. Mice were imaged at 7 and 14 days post transplantation. (g) Overall survival of the mice from the experiment is described in $\mathbf{f}$. Gray shading denotes the period of therapy. The median survival for vehicle and dinaciclib-treated mice were 19 and 26 days, respectively $(P<0.001)$.

recruits $\mathrm{P}-\mathrm{TEFb}$ to its targets as a means to activate Pol II. ${ }^{3,5,6}$ More recently, CDK9-mediated transcriptional elongation was reported as essential for tumor maintenance in a genetically defined MYC-driven model of hepatocellular carcinoma. ${ }^{7}$ Thus, CDK9 dependence may represent a druggable vulnerability in lymphomas with dysregulated MYC expression.

Dinaciclib (Merck, Boston, MA, USA) is a novel CDK inhibitor that has reached phase $1 \mathrm{~b} / 2$ of clinical trials for a range of solid-organ malignancies, as well as for myeloma and chronic lymphocytic leukemia.8 We hypothesized that CDK9 inhibition by dinaciclib would represent a rational pharmacologic approach to target the transcription of critical MYC-regulated oncogenic effector proteins. Here we describe durable in vivo responses to dinaciclib in aggressive MYC-driven lymphoma, mediated by downregulation of Pol II-mediated Mcl-1 transcription.

Dinaciclib has $50 \%$ kinase inhibitory concentrations of $1,1,3$ and $4 \mathrm{~nm}$ for CDK2, CDK5, CDK1 and CDK9, respectively. ${ }^{8}$ Dinaciclib potently killed $\mathrm{E} \mu$-Myc and human IG-CMYC-translocated cell lines independent of p53 function, but not untransformed murine fibroblast cells, at low nanomolar concentrations approximating those observed for kinase inhibition (Figures $1 a$ and $b$, Supplementary Figure S1). 
As $\mathrm{BCl}-2$ and $\mathrm{Mcl}-1$ have been implicated as important apoptotic regulators in $\mathrm{E} \mu-M y c$ lymphomas, ${ }^{9,10}$ we assessed the effects of dinaciclib on these proteins. We hypothesized that CDK9 inhibition with dinaciclib would target Mcl-1 transcription, as has been observed with other CDK inhibitors in myeloma and mantle cell lymphoma. ${ }^{11,12}$ E $\mu$-Myc and human IG-CMYC-translocated cell lines were treated with dinaciclib or dimethylsulfoxide control and interrogated using the quantitative PCR analysis for the effect on $\mathrm{Mcl}-1$ and $\mathrm{Bcl}-2$ mRNA. Dinaciclib treatment was associated with a significant reduction in Mcl-1 mRNA, with no significant effect on $\mathrm{BCl}-2$ transcript levels (Figure 1C, Supplementary Figure S2). Chromatin immunoprecipitation-PCR was used to show the binding of phosphorylated Pol II, subunit B1 carboxy-terminal domain (CTD) serine 2 (pRpb1 Ser2) as a marker of CDK9 activity at the $\mathrm{Mcl}-1$ locus in a representative E $\mu-M y c$ lymphoma cell line (Figure 1d). These findings support the hypothesis that dinaciclib transcriptionally downregulates $\mathrm{Mcl}-1$.

We next examined $\mathrm{Mcl}-1$ expression in $\mathrm{E} \mu-\mathrm{Myc}$ and human IGcMYC-translocated lymphoma cell lysates following the treatment with dinaciclib or vehicle. On-target CDK9 inhibition by dinaciclib was confirmed through inhibition of pRpb1 Ser2 at concentrations corresponding to apoptosis induction in E $\mu-M y c$ cells (Figure 1e). Dinaciclib treatment also rapidly suppressed $\mathrm{Mcl}-1$ protein expression, with no discernible reduction in $\mathrm{Bcl}-2$ or $\mathrm{Bcl}-\mathrm{x}_{\mathrm{L}}$ protein observed in murine (Figure 1e) or human (Figure 1f) cells. To determine the functional importance of $\mathrm{Mcl}-1$ in regulating dinaciclib-mediated apoptosis, a representative $\mathrm{E} \mu-M y c$ lymphoma was stably transduced to express $\mathrm{Mcl}-1$ off a retroviral promoter. As shown in Figure 1g, exogenously expressed Mcl-1 significantly protected E $\mu-M y c$ cells from dinaciclib-induced apoptosis.

The in vivo efficacy of dinaciclib was then assessed by transplanting the same $\mathrm{E} \mu-M y c$ lymphomas into cohorts of syngeneic $\mathrm{C} 57 \mathrm{BI} / 6$ recipients. Compared with the vehicle control, dinaciclib treatment was well tolerated and associated with a highly significant survival advantage of tumor-bearing mice, including those bearing a p53-null lymphoma and a lymphoma with a spontaneous p53 mutation encoding a dominant-negative p53 protein (Figures $2 \mathrm{a}-\mathrm{c}$, Supplementary Figure S3). In contrast, dinaciclib-mediated therapeutic efficacy was severely attenuated in isogeneic p53-competent $\mathrm{E} \mu-M y c$ lymphoma overexpressing Mcl-1 (Figure 2d). In separate experiments, mice bearing transplanted E $\mu-M y c$ cells were left untreated for 12 days to establish bulky nodal disease, at which time they received a single dose of dinaciclib or vehicle 1 or $4 \mathrm{~h}$ before being killed and before the lymph nodes were harvested. Consistent with the in vitro data, lymph node protein lysates showed reductions of pRpb1 and total Mcl-1 protein (Figure 2e), concomitant with the induction of apoptosis (Supplementary Figure S4). Finally, dinaciclib treatment of immunocompromised mice xenografted with the human IG-CMYC-translocated lymphoma was associated with reduced disease progression and significantly prolonged overall survival (Figures $2 \mathrm{f}$ and $\mathrm{g}$ ).

In conclusion, our findings indicate that CDK9 inhibition by dinaciclib is highly effective in aggressive MYC-driven lymphomas, including 'poor-risk' p53-deficient clones, via selective inhibition of critical MYC targets including Mcl-1 (which is currently undruggable with existing $\mathrm{BH} 3$ mimetics). ${ }^{13,14}$ Our data suggest a linear and druggable dependency between MYC and Mcl-1 that is contingent on CDK9 signaling. These findings are of particular interest in the context of a recent publication by Kelly et al., ${ }^{15}$ further highlighting the dependency of MYC-driven B-cell lymphoma to $\mathrm{Mcl}-1$. Rapid clinical translation of CDK9 inhibitors to MYC-dysregulated lymphoid malignancy should now be considered.

\section{CONFLICT OF INTEREST}

The authors declare no conflict of interest.

\section{ACKNOWLEDGEMENTS}

Researchers are supported by funding from the Leukaemia Foundation of Australia (GPG, SJH, ML, MAD), the Royal Australasian College of Physicians (GPG) and the Arrow Bone Marrow Transplant Foundation (AB, ML). LMK is supported by a CJ Martin Fellowship (NHMRC), MAD is supported by VESKI Innovation and Herman Clinical Research Fellowships, JS is supported by funding from the Eva and Les Erdi/ Snowdome Foundation Victorian Cancer Agency Fellowship, RWJ is a Principal Research Fellow of the National Health and Medical Research Council of Australia (NHMRC) and is supported by NHMRC Program and Project Grants, the Cance Council Victoria and the Victorian Cancer Agency. Dinaciclib was provided by Merck Research Laboratories (Boston, MA, USA) and a-Amanitin was kindly provided by Ms Christina Woelwer. We thank Mr Don Cameron for technical advice regarding a-Amanitin experiments. The TRMPVIR vector was kindly provided by Dr Johannes Zuber.

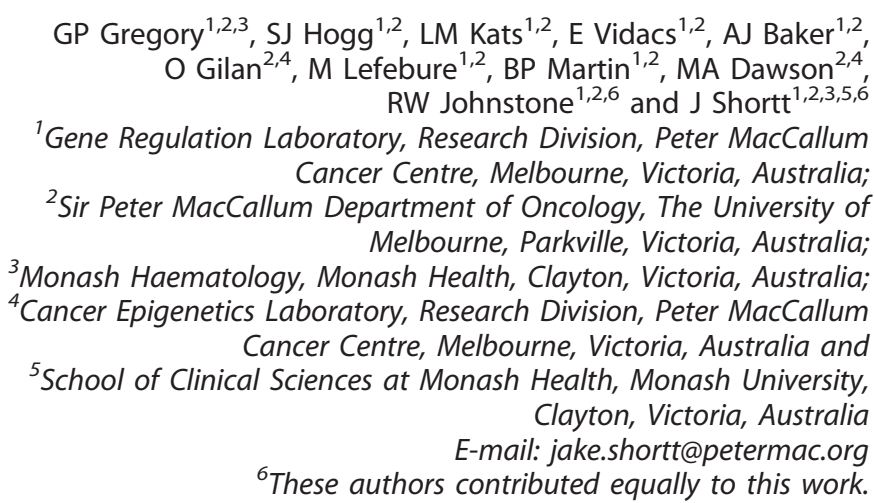

\section{REFERENCES}

1 Savage KJ, Johnson NA, Ben-Neriah S, Connors JM, Sehn LH, Farinha P et al. MYC gene rearrangements are associated with a poor prognosis in diffuse large B-cell lymphoma patients treated with R-CHOP chemotherapy. Blood 2009; 114: 3533-3537.

2 Hu S, Xu-Monette ZY, Tzankov A, Green T, Wu L, Balasubramanyam A et al. MYC/BCL2 protein coexpression contributes to the inferior survival of activated B-cell subtype of diffuse large B-cell lymphoma and demonstrates high-risk gene expression signatures: a report from The International DLBCL Rituximab-CHOP Consortium Program. Blood 2013; 121: 4021-4031.

3 Kanazawa S, Soucek L, Evan G, Okamoto T, Peterlin BM. c-MYC recruits P-TEFb for transcription, cellular proliferation and apoptosis. Oncogene 2003; 22: 5707-5711.

4 Marshall NF, Peng J, Xie Z, Price DH. Control of RNA polymerase II elongation potential by a novel carboxyl-terminal domain kinase. J Biol Chem 1996; 271: 27176-27183.

5 Gargano B, Amente S, Majello B, Lania L. P-TEFb is a crucial co-factor for Myc transactivation. Cell Cycle 2007; 6: 2031-2037.

6 Cowling VH, Cole MD. The Myc transactivation domain promotes global phosphorylation of the RNA polymerase II carboxy-terminal domain independently of direct DNA binding. Mol Cell Biol 2007; 27: 2059-2073.

7 Huang $\mathrm{CH}$, Lujambio A, Zuber J, Tschaharganeh DF, Doran MG, Evans MJ et al. CDK9-mediated transcription elongation is required for MYC addiction in hepatocellular carcinoma. Genes Dev 2014; 28: 1800-1814.

8 Parry D, Guzi T, Shanahan F, Davis N, Prabhavalkar D, Wiswell D et al. Dinaciclib (SCH 727965), a novel and potent cyclin-dependent kinase inhibitor. Mol Cancer Ther 2010; 9: 2344-2353.

9 Adams JM, Harris AW, Pinkert CA, Corcoran LM, Alexander WS, Cory S et al. The c-myc oncogene driven by immunoglobulin enhancers induces lymphoid malignancy in transgenic mice. Nature 1985; 318: 533-538.

10 Mason KD, Vandenberg $\mathrm{CJ}$, Scott $\mathrm{CL}$, Wei AH, Cory S, Huang DCS et al. In vivo efficacy of the $\mathrm{BCl}-2$ antagonist ABT-737 against aggressive Myc-driven lymphomas. Proc Natl Acad Sci USA 2008; 105: 17961-17966.

11 Raje N, Kumar S, Hideshima T, Roccaro A, Ishitsuka K, Yasui H et al. Seliciclib (CYC202 or R-roscovitine), a small-molecule cyclin-dependent kinase inhibitor mediates activity via down-regulation of $\mathrm{Mcl}-1$ in multiple myeloma. Blood 2005; 106: 1042-1047.

12 Lacrima K, Valentini A, Lambertini C, Taborelli M, Rinaldi A, Zucca E et al. In vitro activity of cyclin-dependent kinase inhibitor CYC202 (Seliciclib, R-roscovitine) in mantle cell lymphomas. Ann Oncol 2005; 16: 1169-1176.

13 Oltersdorf T, Elmore SW, Shoemaker AR, Armstrong RC, Augeri DJ, Belli BA et al. An inhibitor of $\mathrm{Bcl}-2$ family proteins induces regression of solid tumours. Nature 2005; 435: 677-681. 
14 Souers AJ, Leverson JD, Boghaert ER, Ackler SL, Catron ND, Chen J et al. ABT-199, a potent and selective $B C L-2$ inhibitor, achieves antitumor activity while sparing platelets. Nat Med 2013; 19: 202-208.

15 Kelly GL, Grabow S, Glaser SP, Fitzsimmons L, Aubrey BJ, Okamoto T et al. Targeting of MCL-1 kills MYC-driven mouse and human lymphomas even when they bear mutations in p53. Genes Dev 2014; 28: 58-70.
This work is licensed under a Creative Commons AttributionBY NC ND NonCommercial-NoDerivs 4.0 International License. The images or
other third party material in this article are included in the article's Creative Commons license, unless indicated otherwise in the credit line; if the material is not included under the Creative Commons license, users will need to obtain permission from the license holder to reproduce the material. To view a copy of this license, visit http:// creativecommons.org/licenses/by-nc-nd/4.0/

\section{Targeting PD1-PDL1 immune checkpoint in plasmacytoid dendritic cell interactions with $\mathrm{T}$ cells, natural killer cells and multiple myeloma cells}

\author{
Leukemia (2015) 29, 1441-1444; doi:10.1038/leu.2015.11
}

Despite the advent of bortezomib, thalidomide and lenalidomide, relapse of multiple myeloma (MM) is common, and novel therapies are needed urgently. ${ }^{1}$ Interactions of MM cells with bone marrow (BM) accessory and immune effector cells inhibit antitumor immunity as well as induce MM growth, survival and drug resistance. ${ }^{1}$ For example, we showed that plasmacytoid dendritic cells ( $\mathrm{pDCs}$ ) are increased in the $\mathrm{BM}$ of MM patients compared with normal $\mathrm{BM}$, and these contribute to immune dysfunction, as well as promote tumor cell growth and survival. ${ }^{2}$ Aberrant pDCs' function in $\mathrm{MM}$ is evidenced by their interaction not only with MM cells but also with immune effector T cells: MM BM pDCs have decreased ability to trigger T-cell proliferation compared with normal pDCs. ${ }^{2}$ Dysfunctional T cells and natural killer (NK) cells in $\mathrm{MM}^{3,4}$ together with functionally defective $\mathrm{pDCs}^{2}$ confer immune suppression in MM. To date, the mechanism(s) and the role of immunoregulatory molecules mediating $\mathrm{pDC}-\mathrm{T}$ cell and $\mathrm{pDC}-\mathrm{NK}$ cell interactions in MM remain undefined. Here we extended our previous studies ${ }^{2,5}$ to examine the role of immune checkpoint receptor programmed cell death protein 1 (PD1) and its ligand PDL1 in pDC-T cell and PDC-NK cell interactions in the MM BM milieu, and to determine whether this interaction represents a therapeutic target to restore antitumor immunity and cytotoxicity.

PD1 (CD279), a member of the CD28 family of receptors, is expressed on the surface of antigen-activated and -exhausted T cells. ${ }^{4}$ PD1 has two ligands, PDL1 (B7-H1; CD274) and PDL2 (B7-DC; CD273). Although PDL1 expression has not been observed in normal epithelial cells, it is highly expressed on many solid tumors. ${ }^{6}$ PDL2 is more broadly expressed on normal healthy tissues than PDL1. The physiological role of PD1 is to maintain T-cell homeostasis by restricting T-cell activation and proliferation, thereby preventing autoimmunity. Importantly, the interaction of PD1+ $T$ cells with PDL1-expressing cells inhibits T-cell responses. $^{7-9}$ In the context of MM, studies have demonstrated PD1-expressing T cells and NK cells in the MM BM milieu, as well as PDL1 on MM cells. ${ }^{3,10-13}$ However, the expression of PDL1-PD1 on $\mathrm{MM}$ patient-derived $\mathrm{pDCs}$ and its functional significance during PDC-MM-T-NK cell interactions remain undefined.

We first analyzed freshly isolated MM cells, $\mathrm{pDCs}$ and T cells from MM patient BM samples $(n=11)$ for PDL1 and PD1 expression using flow cytometry (fluorescence-activated cell sorter (FACS)). Both MM cells and pDCs expressed high surface levels of
PDL1, whereas T cells showed high PD1 levels (Figures 1a-c). No significant PDL1 expression was noted on normal BM plasma cells. Our findings are consistent with previous reports showing that MM cells, but not normal plasma cells, express PDL1, ${ }^{3,10-13}$ These data indicate that the interactions between PDL1-expressing MM cells and pDCs with PD1-positive T cells may contribute to both $\mathrm{T}$-cell and $\mathrm{PDC}$ immune dysfunction in MM, and MM cells may escape antitumor immunity by virtue of PDL1 expression.

We next examined whether blockade of PDL1-PD1 restores anti-MM immune response and/or affects $\mathrm{PDC}$-induced $\mathrm{MM}$ cell growth, using a monoclonal antibody (Ab) specifically directed against PDL1. A recent study analyzed the expression of PD1 and PD1-ligands in the tumor immune microenvironment and demonstrated clinical responses to anti-PD1 Ab therapy in PDL1positive tumors. ${ }^{8}$ PDL1 is expressed in both PDCs and MM cells, including relapsed or refractory $\mathrm{MM}^{13}$ and we hypothesize that blockade of PDL1 will alleviate T-cell immune suppression conferred by both MM cells and pDCs during PDC-MM-T cell interactions. Moreover, as PDL1 binds not only to PD1 but also to CD80, on T cells to induce T-cell inhibition, ${ }^{14}$ anti-PDL1 Ab may block both co-inhibitory signals on T cells. Preclinical and clinical studies have begun to examine the utility of anti-PDL1 monoclonal $A b$ in MM..$^{10,11,15}$ Here we targeted PDL1 rather than PDL2 for the following reasons: (1) PDL1 is more restricted in its expression on normal tissues than PDL2, and targeting PDL1 may therefore cause less on-target off-tissue toxicity; ${ }^{9}$ (2) a recent report correlated PDL1, but not PDL2, expression with response to anti-PD1 therapy; ${ }^{8}$ and (3) we found that both PDCs and MM cells express variable and low levels of PDL2 versus PDL1.

We first examined whether blockade of PDL1 affects the ability of $\mathrm{pDC}$ to induce MM cell growth. The patient MM cells or MM cell lines (MM.1S, MM.1R and RPMI-8226) were cultured either alone or together with MM-pDCs in the presence or absence of anti-PDL1 $\mathrm{Ab}$ for $72 \mathrm{~h}$, followed by analysis of growth. pDCs triggered proliferation of autologous MM cells and MM cell lines, as in our previous studies. ${ }^{2,5}$ Importantly, anti-PDL1 Ab did not significantly inhibit PDC-triggered growth of MM cells (Figure 1d and Supplementary Figure 1). Our recent study showed that targeting toll-like receptor-9 blocks pDC-induced MM cell growth, ${ }^{2,5}$ which served as a positive control in these studies (Figure 1d and Supplementary Figure 1). Although blocking PDL1 does not affect pDC-induced MM cell growth, $\mathrm{PDC}-\mathrm{MM}$ cell interactions upregulate PDL1 expression on both cell types, consistent with earlier observations that BM stromal cells induce PDL1 expression on MM cells. ${ }^{13}$ Such interactive mechanisms enhancing PDL1 expression in the MM BM milieu further abrogate PD1-expressing T-cell 